\title{
Expectation Properties of Generalized Order Statistics from Poisson Lomax Distribution
}

\author{
Haseeb Athar ${ }^{1,2, *}$, Zubdahe Noor ${ }^{1}$, Saima Zarrin ${ }^{1}$, Hanadi N.S. Almutairi ${ }^{2}$ \\ ${ }^{1}$ Department of Statistics and Operations Research, Aligarh Muslim University, Aligarh, India \\ 2 Department of Mathematics, Faculty of Science, Taibah University, Al-Madinah, Kingdom of Saudi Arabia
}

\begin{abstract}
The Poisson Lomax distribution was proposed by [3], as a useful model for analyzing lifetime data. In this paper, we have derived recurrence relations for single and product moments of generalized order statistics for this distribution. Further, characterization of the distribution is carried out. Some deductions and particular cases are also discussed.
\end{abstract}

Keywords Poisson Lomax Distribution; Generalized Order Statistics; Single Moments; Product Moments; Characterization.

AMS 2010 subject classifications 62G30, 62E10, 62E05

\section{DOI:10.19139/soic-2310-5070-614}

\section{Introduction}

The Poisson Lomax distribution was proposed by [3], as a three-parameter lifetime distribution with upside-down bathtub shaped failure rate and heavy tailed, and can be used in modelling many practical situation. It is a compound distribution of the zero-truncated Poisson and the Lomax distributions.

A random variable $X$ is said to follow the Poisson Lomax distribution (PLD) if its probability density function $(p d f)$ is of the form

$$
f(x)=\frac{\alpha \beta \lambda(1+\beta x)^{-(1+\alpha)} e^{-\lambda(1+\beta x)^{-\alpha}}}{1-e^{-\lambda}}, \quad x>0, \alpha>0, \beta>0, \lambda>0,
$$

and the corresponding survival function is

$$
\bar{F}(x)=\frac{1-e^{-\lambda(1+\beta x)^{-\alpha}}}{1-e^{-\lambda}}, \quad x>0, \alpha>0, \beta>0, \lambda>0,
$$

where $\bar{F}(x)=1-F(x)$.

In view of (1) and (2), it can be seen that

$$
f(x)=c_{1}(1+\beta x)^{-(\alpha+1)}-c_{2}(1+\beta x)^{-(\alpha+1)} \bar{F}(x) .
$$

where $c_{1}=\frac{\alpha \beta \lambda}{1-e^{-\lambda}}$ and $c_{2}=\alpha \beta \lambda$.

\footnotetext{
*Correspondence to: Haseeb Athar (Email: haseebathar@ hotmail.com).
}

ISSN 2310-5070 (online) ISSN 2311-004X (print)

Copyright (C) 2021 International Academic Press 
Relation (3) can also be expressed as

$$
\frac{\bar{F}(x)}{f(x)}=\frac{1}{\alpha \beta \lambda} \sum_{a=0}^{\infty} \sum_{b=0}^{[\alpha(1-a)+1)]} \frac{\lambda^{a}}{a !}\left(\begin{array}{c}
[\alpha(1-a)+1)] \\
b
\end{array}\right) \beta^{b} x^{b}
$$

where $[\alpha(1-a)+1)]$ is an integer.

\subsection{Generalized order statistics}

The concept of generalized order statistics was introduced and extensively studied by [17], which includes different ordered random schemes, such as order statistics, record values, sequential order statistics, progressively type II censored order statistics and Pfeifer's records as its special cases.

Let $n \geq 2$ be a given integer and $\tilde{m}=\left(m_{1}, m_{2}, \ldots, m_{n-1}\right) \in \mathbb{R}^{n-1}, k \geq 1$ be the parameters, such that

$$
\gamma_{i}=k+n-i+\sum_{j=i}^{n-1} m_{j} \geq 0 \text { for } 1 \leq i \leq n-1 .
$$

The random variables $X(1, n, \tilde{m}, k), X(2, n, \tilde{m}, k), \ldots, X(n, n, \tilde{m}, k)$ are said to be generalized order statistics from an absolutely continuous distribution function $F()$ with the probability density function $(p d f) f()$, if their joint $p d f$ is of the form

$$
k\left(\prod_{j=1}^{n-1} \gamma_{j}\right)\left(\prod_{i=1}^{n-1}\left[1-F\left(x_{i}\right)\right]^{m_{i}} f\left(x_{i}\right)\right)\left[1-F\left(x_{n}\right)\right]^{k-1} f\left(x_{n}\right)
$$

on the cone $F^{-1}(0)<x_{1} \leq x_{2} \leq \ldots \leq x_{n}<F^{-1}(1)$.

If $m_{i}=0 ; i=1 \ldots n-1, k=1$, we obtain the joint $p d f$ of the order statistics and for $m_{i} \rightarrow-1, k \in N$, we get joint $p d f$ of $k^{\text {th }}$ upper record values.

Here we may consider two cases:

Case I. $\gamma_{i} \neq \gamma_{j}, i, j=1,2, \ldots, n-1, i \neq j$.

In view of (5), the $p d f$ of $r^{t h}$ gos $X(r, n, \tilde{m}, k)$ is given as ([18])

$$
f_{X(r, n, \tilde{m}, k)}(x)=C_{r-1} f(x) \sum_{i=1}^{r} a_{i}(r)[\bar{F}(x)]^{\gamma_{i}-1},
$$

where

$$
C_{r-1}=\prod_{i=1}^{r} \gamma_{i}, \quad \gamma_{i}=k+n-i+\sum_{j=1}^{n-1} m_{j}>0
$$

and

$$
a_{i}(r)=\prod_{\substack{j=1 \\ j \neq i}}^{r} \frac{1}{\left(\gamma_{j}-\gamma_{i}\right)}, \quad 1 \leq i \leq r \leq n
$$

The joint $p d f$ of $X(r, n, \tilde{m}, k)$ and $X(s, n, \tilde{m}, k), 1 \leq r<s \leq n$, is given as ([18])

$$
f_{X(r, n, \tilde{m}, k), X(s, n, \tilde{m}, k)}(x, y)=C_{s-1} \sum_{j=r+1}^{s} a_{j}^{(r)}(s)\left(\frac{\bar{F}(y)}{\bar{F}(x)}\right)^{\gamma_{j}}\left[\sum_{i=1}^{r} a_{i}(r)[\bar{F}(x)]^{\gamma_{i}}\right] \frac{f(x)}{\bar{F}(x)} \frac{f(y)}{\bar{F}(y)}, x<y
$$


where

$$
a_{i}{ }^{(r)}(s)=\prod_{\substack{I=r+1 \\ I \neq j}}^{s} \frac{1}{\left(\gamma_{I}-\gamma_{j}\right)}, \quad r+1 \leq j \leq s \leq n .
$$

Case II : $m_{i}=m, i=1,2, \ldots, n-1$.

The $p d f$ of $r^{t h}$ gos $X(r, n, m, k)$ is given as ([17])

where

$$
f_{X(r, n, m, k)}(x)=\frac{C_{r-1}}{(r-1) !}[\bar{F}(x)]^{\gamma_{r}-1} f(x) g_{m}^{r-1}(F(x)),
$$

$$
\begin{aligned}
& C_{r-1}=\prod_{i=1}^{r} \gamma_{i} \quad, \gamma_{i}=k+(n-i)(m+1), \\
& h_{m}(x)= \begin{cases}-\frac{1}{m+1}(1-x)^{m+1} & , m \neq-1 \\
\log \left(\frac{1}{1-x}\right) & , m=-1\end{cases}
\end{aligned}
$$

and

$$
g_{m}(x)=h_{m}(x)-h_{m}(0)=\int_{0}^{x}(1-t)^{m} d t, x \in[0,1) .
$$

The joint $p d f$ of $X(r, n, m, k)$ and $X(s, n, m, k), 1 \leq r<s \leq n$, is given as ([17])

$$
\begin{gathered}
f_{X(r, n, m, k), X(s, n, m, k)}(x, y)=\frac{C_{s-1}}{(r-1) !(s-r-1) !}[\bar{F}(x)]^{m} g_{m}^{r-1}(F(x))\left[h_{m}(F(y))-h_{m}(F(x))\right]^{s-r-1} \\
\times[\bar{F}(y)]^{\gamma_{s}-1} f(x) f(y), \quad-\infty \leq x<y \leq \infty .
\end{gathered}
$$

Also from [23], for $m_{i}=m \neq-1, i=1,2, \ldots, n-1$.

$$
\begin{gathered}
a_{i}(r)=\frac{(-1)^{r-i}}{(m+1)^{r-1}(r-1) !}\left(\begin{array}{c}
r-1 \\
i
\end{array}\right) \\
a_{i}^{(r)}(s)=\frac{(-1)^{s-i}}{(m+1)^{s-r-1}(r-1) !}\left(\begin{array}{c}
s-r-1 \\
i
\end{array}\right)
\end{gathered}
$$

A large volume of work has been done on the study of moments and recurrence relations between moments of generalized order statistics. The moments of ordered random schemes assume considerable importance in the statistical literature. Many authors have investigated and derived several recurrence relations and identities satisfied by the single as well as product moments. [24],[25] studied the recurrence relations and identities for moments of order statistics for some specific distributions. Recurrence relations for the expected values of certain functions of order statistics are considered by [1], [2]. [7] investigated the relations between expected values of functions of gos. For more detailed survey, one may refer to [4], [5], [8], [12], [15], [18], [19], [26], [27], [29], [30], [32] and references therein.

The characterization of a probability distribution has always been the important topic in statistics and mathematical sciences. Several approaches are available to characterize a probability distribution. In this paper, first we established recurrence relations between single and product moments of gos from PLD. Then, these relation are used to characterize the said distribution. Also a characterization theorem based on conditional expectation is presented. For related results on characterization, one can see [6], [9], [10], [11], [13], [20], [22] and [28] among others. 


\subsection{Gauss hypergeometric function}

Gauss hypergeometric function is defined as

$$
{ }_{2} F_{1}(a, b ; c ; x)=\sum_{k=0}^{\infty} \frac{(a)_{k}(b)_{k}}{(c)_{k}} \frac{x^{k}}{k !}
$$

where $c \neq 0,-1,-2, \ldots$ It converges if one of the following conditions holds:

(i) $|x|<1$

(ii) $|x|=1, \operatorname{Re}(c-a-b)>0$.

\section{Single Moments}

Theorem 1

Let case I be satisfied. For the PLD given in (1) and for $n \in N, \tilde{m} \in \mathbb{R}, k>0,1 \leq r \leq n, p=1,2, \ldots$

$$
E\left[X^{p}(r, n, \tilde{m}, k)\right]=\alpha C_{r-1} \sum_{i=1}^{r} \sum_{j=0}^{\gamma_{i}-1} \sum_{l=0}^{\infty} a_{i}(r)\left(\begin{array}{c}
\gamma_{i}-1 \\
j
\end{array}\right) \frac{(-1)^{j+l} \lambda^{l}(j+1)^{l}}{\beta^{p}\left(1-e^{-\lambda}\right)^{\gamma_{i}} l !} \mathbf{B}(p+1, \alpha+\alpha l-p),
$$

where $B(x, y)$ is complete beta function.

Proof

We have

$$
\begin{aligned}
& E\left[X^{p}(r, n, \tilde{m}, k)\right]=C_{r-1} \int_{0}^{\infty} x^{p} \sum_{i=1}^{r} a_{i}(r)[\bar{F}(x)]^{\gamma_{i}-1} f(x) \mathrm{d} x \\
& =C_{r-1} \sum_{i=1}^{r} a_{i}(r) \int_{0}^{\infty} x^{p}[\bar{F}(x)]^{\gamma_{i}-1} \\
& \times\left\{\frac{\alpha \beta \lambda(1+\beta x)^{-(\alpha+1)}}{1-e^{-\lambda}}-\alpha \beta \lambda(1+\beta x)^{-(\alpha+1)} \bar{F}(x)\right\} \mathrm{d} x \\
& =\alpha \beta \lambda C_{r-1} \sum_{i=1}^{r} \frac{a_{i}(r)}{\left(1-e^{-\lambda}\right)^{\gamma_{i}}} \int_{0}^{\infty} x^{p}(1+\beta x)^{-(\alpha+1)} \\
& \times\left\{1-e^{-\lambda(1+\beta x)^{-\alpha}}\right\}^{\gamma_{i}-1} e^{-\lambda(1+\beta x)^{-\alpha}} \mathrm{d} x \\
& =\alpha \beta \lambda C_{r-1} \sum_{i=1}^{r} \frac{a_{i}(r)}{\left(1-e^{-\lambda}\right)^{\gamma_{i}}} \sum_{j=0}^{\gamma_{i}-1}\left(\begin{array}{c}
\gamma_{i}-1 \\
j
\end{array}\right)(-1)^{j} \sum_{l=0}^{\infty} \frac{(-1)^{l} \gamma^{l}(j+1)^{l}}{l !} \\
& \times \int_{0}^{\infty} x^{p}(1+\beta x)^{-(\alpha+\alpha l+1)} \mathrm{d} x .
\end{aligned}
$$

Now, by using the result from [14] $p .315$ given as,

$$
\int_{0}^{\infty} x^{\mu-1}(1+\beta x)^{-\nu} \mathrm{d} x=\beta^{-\mu} \mathbf{B}(\mu, \nu-\mu)
$$

we get

$$
E\left[X^{p}(r, n, \tilde{m}, k)\right]=\alpha C_{r-1} \sum_{i=1}^{r} \sum_{j=0}^{\gamma_{i}-1} \sum_{l=0}^{\infty}\left(\begin{array}{c}
\gamma_{i}-1 \\
j
\end{array}\right) \frac{a_{i}(r) \lambda^{l}(j+1)^{l}(-1)^{j+l}}{\beta^{p}\left(1-e^{-\lambda}\right)^{\gamma_{i}} l !} \mathbf{B}(p+1, \alpha+\alpha l-p) .
$$


Hence the theorem.

\section{Corollaryl}

When $m_{i}=m, i=1,2, \ldots, n-1$, relation (13) reduces to the single moment of $g o s$ for Case-II.

$$
\begin{aligned}
E\left[X^{p}(r, n, m, k)\right]=\alpha C_{r-1} \sum_{i=1}^{r} \sum_{j=0}^{\gamma_{i}-1} \sum_{l=0}^{\infty}\left(\begin{array}{c}
\gamma_{i}-1 \\
j
\end{array}\right)\left(\begin{array}{c}
r-1 \\
i
\end{array}\right) & \\
& \times \frac{(-1)^{r-i+j+l} \lambda^{l+1}(j+1)^{l}}{(r-1) !(m+1)^{r-1} \beta^{p}\left(1-e^{-\lambda}\right)^{\gamma_{i}} l !} \mathbf{B}(p+1, \alpha+\alpha l-p) .
\end{aligned}
$$

\section{Theorem2}

For the conditions as stated in Theorem 1. The recurrence relation for single moments of gos for PLD is given as

$$
\begin{aligned}
E\left[X^{p}(r, n, \tilde{m}, k)\right]-E\left[X^{p}(r-1, n, \tilde{m}, k)\right]=p C_{r-2} \sum_{i=1}^{r} \sum_{j=0}^{\gamma_{i}} \sum_{l=0}^{\infty}\left(\begin{array}{c}
\gamma_{i} \\
j
\end{array}\right) & \\
& \times \frac{a_{i}(r)(-1)^{j+l} \lambda^{l} j^{l}}{l ! \beta^{p}\left(1-e^{-\lambda}\right)^{\gamma_{i}}} \mathbf{B}(p, \alpha l-p) .
\end{aligned}
$$

Also,

$$
\begin{aligned}
& E\left[X^{p}(r, n, \tilde{m}, k)\right]-E\left[X^{p}(r-1, n, \tilde{m}, k)\right]= \\
& \frac{p}{\gamma_{r} \alpha \beta \lambda} \sum_{a=0}^{\infty} \sum_{b=1}^{[\alpha(1-a)+1]}\left(\begin{array}{c}
\alpha(1-a)+1] \\
b
\end{array}\right) \frac{\lambda^{a} \beta^{b}}{a !} E\left[X^{p+b-1}(r, n, \tilde{m}, k)\right] .
\end{aligned}
$$

\section{Proof}

We have by [7].

$$
E[\xi\{X(r, n, \tilde{m}, k)\}]-E[\xi\{X(r-1, n, \tilde{m}, k)\}]=C_{r-2} \int_{-\infty}^{\infty} \xi^{\prime}(x) \sum_{i=1}^{r} a_{i}(r)[\bar{F}(x)]^{\gamma_{i}} \mathrm{~d} x .
$$

For $\xi(x)=x^{p}$ in (19), recurrence relation for single moments of gos is

$$
\begin{aligned}
E\left[X^{p}(r, n, \tilde{m}, k)\right]-E[ & \left.X^{p}(r-1, n, \tilde{m}, k)\right] \\
& =p C_{r-2} \int_{0}^{\infty} x^{p-1} \sum_{i=1}^{r} a_{i}(r)[\bar{F}(x)]^{\gamma_{i}} \mathrm{~d} x . \\
& =p C_{r-2} \sum_{i=1}^{r} a_{i}(r) \int_{0}^{\infty} x^{p-1}\left\{\frac{1-e^{-\lambda(1+\beta x)^{-\alpha}}}{1-e^{-\lambda}}\right\}^{\gamma_{i}} \mathrm{~d} x . \\
& =p C_{r-2} \sum_{i=1}^{r} \sum_{j=0}^{\gamma_{i}} \sum_{l=0}^{\infty}\left(\begin{array}{c}
\gamma_{i} \\
j
\end{array}\right) \frac{a_{i}(r)(-1)^{j+l} \lambda^{l} j^{l}}{l !\left(1-e^{-\lambda}\right)^{\gamma_{i}}} \int_{0}^{\infty} x^{p-1}(1+\beta x)^{-\alpha l} \mathrm{~d} x .
\end{aligned}
$$

Now on simplification of (20) and using (14) we get the required result (17).

The expression (18) can be proved in view of [7] using (4). 


\section{Corollary2}

When $m_{i}=m, i=1,2, \ldots, n-1$, relation (17) and (18) reduces to the single moment of gos for Case-II.

$$
\begin{aligned}
E\left[X^{p}(r, n, m, k)\right]-E\left[X^{p}(r-1, n, m, k)\right]=p C_{r-2} & \sum_{i=1}^{r} \sum_{j=0}^{\gamma_{i}} \sum_{l=0}^{\infty}\left(\begin{array}{c}
\gamma_{i} \\
j
\end{array}\right)\left(\begin{array}{c}
r-1 \\
i
\end{array}\right) \\
& \times \frac{(-1)^{j+l+r-i} \lambda^{l} j^{l}}{(m+1)^{r-1}(r-1) ! l ! \beta^{p}\left(1-e^{-\lambda}\right)^{\gamma_{i}}} \mathbf{B}(p, \alpha l-p) .
\end{aligned}
$$

Also

$$
\begin{aligned}
& E\left[X^{p}(r, n, m, k)\right]-E\left[X^{p}(r-1, n, m, k)\right]= \\
& \frac{p}{\gamma_{r} \alpha \beta \lambda} \sum_{a=0}^{\infty} \sum_{b=1}^{[\alpha(1-a)+1]}\left(\begin{array}{c}
{[\alpha(1-a)+1]} \\
b
\end{array}\right) \frac{\lambda^{a} \beta^{b}}{a !} E\left[X^{p+b-1}(r, n, m, k)\right] .
\end{aligned}
$$

\section{Remark1}

Putting $m_{i}=0, i=1,2, \ldots, n-1$ and $k=1$, we get the recurrence relation for single moments of order statistics

$$
E\left[X_{r: n}^{p}\right]-E\left[X_{r-1: n}^{p}\right]=\frac{p}{(n-r-1) \alpha \beta \lambda} \sum_{a=0}^{\infty} \sum_{b=1}^{[\alpha(1-a)+1]}\left(\begin{array}{c}
\alpha(1-a)+1] \\
b
\end{array}\right) \frac{\lambda^{a} \beta^{b}}{a !} E\left[X_{r: n}^{p+b-1}\right]
$$

\section{Remark2}

When $m_{i} \rightarrow-1, i=1,2, \ldots, n-1$, the recurrence relation for single moments of $k^{t h}$ upper record values will be

$$
E\left(X_{U(r)}^{(k)}\right)^{p}-E\left(X_{U(r-1)}^{(k)}\right)^{p}=\frac{p}{k \alpha \beta \lambda} \sum_{a=0}^{\infty} \sum_{b=1}^{[\alpha(1-a)+1]}\left(\begin{array}{c}
{[\alpha(1-a)+1]} \\
b
\end{array}\right) \frac{\lambda^{a} \beta^{b}}{a !} E\left(X_{U(r)}^{(k)}\right)^{p+b-1} .
$$

\section{Product Moments}

\section{Theorem 3}

Let case-I be satisfied For the Poisson Lomax distribution given as in (1) and for $n \in N, \tilde{m} \in \mathbb{R}, k>0,1 \leq r<$ $s \leq n, p, q=1,2, \ldots$, then the $(p, q)$ th product moment is given by

$$
\begin{aligned}
\mu_{r, s, n, \tilde{m}, k}^{p, q}=\alpha^{2} C_{s-1} \sum_{i=1}^{r} \sum_{j=r+1}^{s} \sum_{l=0}^{\gamma_{j}-1} \sum_{u=0}^{\infty} \sum_{v=0}^{\gamma_{i}-\gamma_{j}-1} \sum_{w=0}^{\infty} a_{i}(r) a_{j}^{(r)}(s) \\
\times\left(\begin{array}{c}
\gamma_{j}-1 \\
l
\end{array}\right)\left(\begin{array}{c}
\gamma_{i}-\gamma_{j}-1 \\
v
\end{array}\right) \frac{(\alpha+\alpha t+1)_{u}(\alpha+\alpha t-q)_{w}}{u !(\alpha+\alpha t-q+1)_{u}} \\
\times \frac{(-1)^{l+t+v+w+u} \lambda^{t+w+2}(l+1)^{t}(v+1)^{w}}{t !\left(1-e^{-\lambda}\right)^{\gamma_{i}} \beta^{p+q-1}(\alpha+\alpha t-q)}
\end{aligned}
$$

Proof

We have

$$
\begin{aligned}
\mu_{r, s, n, \tilde{m}, k}^{p, q} & =E\left[X^{p}(r, n, \tilde{m}, k), X^{q}(s, n, \tilde{m}, k)\right] \\
& =C_{s-1} \sum_{i=1}^{r} a_{i}(r) \sum_{j=r+1}^{s} a_{j}^{(r)}(s) \int_{0}^{\infty} \int_{x}^{\infty} x^{p} y^{q}\left[\frac{\bar{F}(y)}{\bar{F}(x)}\right]^{\gamma_{j}}[\bar{F}(x)]^{\gamma_{i}} \frac{f(x)}{\bar{F}(x)} \frac{f(y)}{\bar{F}(y)} \mathrm{d} x \mathrm{~d} y
\end{aligned}
$$




$$
\begin{aligned}
& =C_{s-1} \sum_{i=1}^{r} a_{i}(r) \sum_{j=r+1}^{s} a_{j}^{(r)}(s) \int_{0}^{\infty} \int_{x}^{\infty} x^{p} y^{q}[\bar{F}(y)]^{\gamma_{j}-1}[\bar{F}(x)]^{\gamma_{i}-\gamma_{j}-1} f(x) f(y) \mathrm{d} x \mathrm{~d} y \\
= & C_{s-1} \sum_{i=1}^{r} a_{i}(r) \sum_{j=r+1}^{s} a_{j}^{(r)}(s) \int_{0}^{\infty} x^{p}[\bar{F}(x)]^{\gamma_{i}-\gamma_{j}-1} f(x) \mathrm{d} x \int_{x}^{\infty} y^{q}[\bar{F}(y)]^{\gamma_{j}-1} f(y) \mathrm{d} y .
\end{aligned}
$$

Consider

$$
\begin{aligned}
& I(y)=\int_{x}^{\infty} y^{q}[\bar{F}(y)]^{\gamma_{j}-1} f(y) \mathrm{d} y \\
& \quad=\int_{x}^{\infty} y^{q}[\bar{F}(y)]^{\gamma_{j}-1}\left\{\frac{\alpha \beta \lambda(1+\beta y)^{-(\alpha+1)}}{1-e^{-\lambda}}-\alpha \beta \lambda(1+\beta y)^{-(\alpha+1)} \bar{F}(y)\right\} \mathrm{d} y \\
& =\frac{\alpha \beta \lambda}{\left(1-e^{-\lambda}\right)^{\gamma_{i}}} \int_{x}^{\infty} y^{q}(1+\beta y)^{-(\alpha+1)}\left[1-e^{-\lambda(1+\beta y)^{-\alpha}}\right]^{\gamma_{j}-1} e^{-\lambda(1+\beta y)^{-\alpha}} \mathrm{d} y \\
& =\frac{\alpha \beta \lambda}{\left(1-e^{-\lambda}\right)^{\gamma_{i}}} \sum_{l=0}^{\gamma_{j}-1}\left(\begin{array}{c}
\gamma_{j}-1 \\
l
\end{array}\right) \sum_{t=0}^{\infty} \frac{(-1)^{l+t} \lambda^{t}(l+1)^{t}}{t !} \times \int_{x}^{\infty} y^{q}(1+\beta y)^{-(\alpha+\alpha t+1)} \mathrm{d} y .
\end{aligned}
$$

Since we have from [14], p. 315.

$$
\int_{u}^{\infty} x^{\mu-1}(1+\beta x)^{-\nu} \mathrm{d} x=\frac{u^{\mu-\nu}}{\beta^{\nu}(\mu-\nu)}{ }_{2} F_{1}\left(\nu, \nu-\mu ; \nu-\mu+1 ;-\frac{1}{\beta u}\right) .
$$

Thus using (26) and (12) in (25), we get

$$
\begin{aligned}
& I(y)=\frac{\alpha}{\left(1-e^{-\lambda}\right)^{\gamma_{i}}} \sum_{l=0}^{\gamma_{j}-1}\left(\begin{array}{c}
\gamma_{j}-1 \\
l
\end{array}\right) \sum_{t=0}^{\infty} \frac{(-1)^{l+t} \lambda^{t+1}(l+1)^{t}}{t !} \\
& \times \frac{x^{q-\alpha-\alpha t}}{\beta^{\alpha+\alpha t}(q-\alpha-\alpha t)}{ }_{2} F_{1}\left(\alpha+\alpha t+1, \alpha+\alpha t-q ; \alpha+\alpha t+1 ;-\frac{1}{\beta x}\right) . \\
& I(y)=\frac{\alpha}{\left(1-e^{-\lambda}\right)^{\gamma_{i}}} \sum_{l=0}^{\gamma_{j}-1}\left(\begin{array}{c}
\gamma_{j}-1 \\
l
\end{array}\right) \sum_{t=0}^{\infty} \sum_{u=0}^{\infty} \frac{(-1)^{l+t+u} \lambda^{t+1}(l+1)^{t} x^{q-\alpha-\alpha t-1}}{t ! u ! \beta^{\alpha+\alpha t}(q-\alpha-\alpha t)} \\
& \quad \times \frac{(\alpha+\alpha t+1)_{u}(\alpha+\alpha t-q)_{u}}{(q-\alpha-\alpha t-q+1)_{u}} .
\end{aligned}
$$

Now using (27) in (24), we get

$$
\begin{aligned}
& \mu_{r, s, n, \tilde{m}, k}^{p, q}=\alpha C_{s-1} \sum_{i=1}^{r} \frac{a_{i}(r)}{\left(1-e^{-\lambda}\right)^{\gamma_{i}}} \sum_{j=r+1}^{s} a_{j}^{(r)}(s) \sum_{l=0}^{\gamma_{j}-1}\left(\begin{array}{c}
\gamma_{j}-1 \\
l
\end{array}\right) \\
& \times \sum_{t=0}^{\infty} \sum_{u=0}^{\infty} \frac{(-1)^{l+t+u} \lambda^{t+1}(l+1)^{t}}{t ! u ! \beta^{\alpha+\alpha t}(q-\alpha-\alpha t)} \frac{(\alpha+\alpha t+1)_{u}(\alpha+\alpha t-q)_{u}}{(q-\alpha-\alpha t-q+1)_{u}} \\
& \quad \times \int_{0}^{\infty} x^{p+q-\alpha-\alpha t-u}[\bar{F}(x)]^{\gamma_{i}-\gamma_{j}-1} f(x) \mathrm{d} x .
\end{aligned}
$$

Again consider the integral of (28) as

$$
I(x)=\int_{0}^{\infty} x^{p+q-\alpha-\alpha t-u}[\bar{F}(x)]^{\gamma_{i}-\gamma_{j}-1} f(x) \mathrm{d} x .
$$




$$
\begin{gathered}
=\int_{0}^{\infty} x^{p+q-\alpha-\alpha t-u}[\bar{F}(x)]^{\gamma_{i}-\gamma_{j}-1}\left\{\frac{\alpha \beta \lambda(1+\beta x)^{-(\alpha+1)}}{1-e^{-\lambda}}-\alpha \beta \lambda(1+\beta x)^{-(\alpha+1)} \bar{F}(x)\right\} \mathrm{d} x \\
=\frac{\alpha \beta \lambda}{\left(1-e^{-\lambda}\right)^{\gamma_{i}-\gamma_{j}}} \int_{0}^{\infty} x^{p+q-\alpha-\alpha t-u}(1+\beta x)^{-(\alpha+1)} e^{-\lambda(1+\beta x)^{-\alpha}}\left(1-e^{-\lambda(1+\beta x)^{-\alpha}}\right)^{\gamma_{i}-\gamma_{j}-1} \mathrm{~d} x \\
=\frac{\alpha \beta \lambda}{\left(1-e^{-\lambda}\right)^{\gamma_{i}-\gamma_{j}}} \sum_{v=0}^{\gamma_{i}-\gamma_{j}-1}\left(\begin{array}{c}
\gamma_{i}-\gamma_{j}-1 \\
v
\end{array}\right) \sum_{w=0}^{\infty} \frac{(-1)^{v+w} \lambda^{w}(v+1)^{w}}{w !} \\
\quad \times \int_{0}^{\infty} x^{p+q-\alpha-\alpha t-u}(1+\beta x)^{-(\alpha+\alpha t+1)} \mathrm{d} x \\
=\frac{\alpha \beta \lambda}{\left(1-e^{-\lambda}\right)^{\gamma_{i}-\gamma_{j}}} \sum_{v=0}^{\gamma_{i}-\gamma_{j}-1}\left(\begin{array}{c}
\gamma_{i}-\gamma_{j}-1 \\
v
\end{array}\right) \sum_{w=0}^{\infty} \frac{(-1)^{v+w} \lambda^{w}(v+1)^{w}}{w ! \beta p+q-u-\alpha-\alpha t+1} \\
\times \mathbf{B}(p+q-u-\alpha-\alpha t+1, \alpha(t+w+2)-p-q+u)
\end{gathered}
$$

Now using (29) in (28), we get the required result.

\section{Corollary3}

When $m_{i}=m, i=1,2, \ldots, n-1$, relation (23) reduces to the product moment of $g o s$ for Case-II.

$$
\begin{aligned}
\mu_{r, s, n, m, k}^{p, q}=\alpha^{2} C_{s-1} \sum_{i=1}^{r} \sum_{j=r+1}^{s} \sum_{l=0}^{\gamma_{j}-1} \sum_{u=0}^{\infty} \sum_{v=0}^{\gamma_{i}-\gamma_{j}-1} \sum_{w=0}^{\infty}\left(\begin{array}{c}
r-1 \\
i
\end{array}\right)\left(\begin{array}{c}
s-r-1 \\
j
\end{array}\right) \\
\times\left(\begin{array}{c}
\gamma_{j}-1 \\
l
\end{array}\right)\left(\begin{array}{c}
\gamma_{i}-\gamma_{j}-1 \\
v
\end{array}\right) \frac{(\alpha+\alpha t+1)_{u}(\alpha+\alpha t-h)_{w}}{u !(\alpha+\alpha t-q+1)_{u}} \\
\times \frac{(-1)^{r+s-i-j l+t+v+w+u} \lambda^{t+w+2}(l+1)^{t}(v+1)^{w}}{(m+1)^{s-2}(r-1) ! 2 t !\left(1-e^{-\lambda}\right)^{\gamma_{i}} \beta^{p+q-1}(\alpha+\alpha t-q)}
\end{aligned}
$$

Theorem4

Under the condition as stated in Theorem 3 The recurrence relation for product moments is given as

$$
\begin{aligned}
& E\left[X^{p}(r, n, \tilde{m}, k) X^{q}(s, n, \tilde{m}, k)\right]-E\left[X^{p}(r, n, \tilde{m}, k) X^{q}(s-1, n, \tilde{m}, k)\right] \\
& =q \alpha C_{s-1} \sum_{i=1}^{r} a_{i}(r) \sum_{j=r+1}^{s} a_{j}^{(r)}(s) \sum_{l=0}^{\gamma_{j}} \sum_{c=0}^{\infty} \sum_{d=0}^{\infty} \sum_{t=0}^{\gamma_{i}-\gamma_{j}-1} \sum_{u=0}^{\infty} \\
& \times \frac{\left(\begin{array}{c}
\gamma_{j} \\
l
\end{array}\right)\left(\begin{array}{c}
\gamma_{i}-\gamma_{j}-1 \\
t
\end{array}\right)}{\left(1-e^{-\lambda}\right)^{\gamma_{i}}} \frac{(\alpha c)_{d}(\alpha c-q)_{d}}{(\alpha c-q+1)_{d}} \times \frac{\lambda^{c+u+1} l^{c}(t+1)^{u}(-1)^{l+c+d+t+q}}{u ! d ! \beta^{p+q}(\alpha c-q)} \\
& \times \mathbf{B}(p+\alpha c-d+1 ; \alpha-\alpha u-p-q+\alpha c+d) .
\end{aligned}
$$

Also,

$$
\begin{aligned}
& E\left[X^{p}(r, n, \tilde{m}, k) X^{q}(s, n, \tilde{m}, k)\right]-E\left[X^{p}(r, n, \tilde{m}, k) X^{q}(s-1, n, \tilde{m}, k)\right] \\
& =\frac{q}{\gamma_{s} \alpha \beta \lambda} \sum_{a=0}^{\infty} \sum_{b=1}^{[\alpha(1-a)+1]}\left(\begin{array}{c}
{[\alpha(1-a)+1]} \\
b
\end{array}\right) \frac{\lambda^{a} \beta^{b}}{a !} E\left[X^{p}(r, n, \tilde{m}, k) X^{q+b-1}(s, n, \tilde{m}, k)\right] .
\end{aligned}
$$

Proof

We have by [7], 


$$
\begin{aligned}
& E[\xi\{X(r, n, \tilde{m}, k), X(s, n, \tilde{m}, k)\}]-E[\xi\{X(r, n, \tilde{m}, k), X(s-1, n, \tilde{m}, k)\}] \\
& \quad=q C_{s-1} \int_{-\infty}^{\infty} \int_{x}^{\infty} \frac{\partial}{\partial y} \xi(x, y) \sum_{j=r+1}^{s} a_{j}^{(r)}(s)\left[\frac{\bar{F}(y)}{\bar{F}(x)}\right]^{\gamma_{j}} \sum_{i=1}^{r} a_{i}(r)[\bar{F}(x)]^{\gamma_{i}} \frac{f(x)}{\bar{F}(x)} d y d x .
\end{aligned}
$$

Let $\xi(x, y)=\xi_{1}(x) \xi_{2}(y)=x^{p} y^{q}$, we get

$$
\begin{aligned}
& E\left[X^{p}(r, n, \tilde{m}, k) X^{q}(s, n, \tilde{m}, k)\right]-E\left[X^{p}(r, n, \tilde{m}, k) X^{q}(s-1, n, \tilde{m}, k)\right] \\
& \quad=q C_{s-1} \sum_{i=1}^{r} a_{i}(r) \sum_{j=r+1}^{s} a_{j}^{(r)}(s) \int_{0}^{\infty} x^{p}[\bar{F}(x)]^{\gamma_{i}-\gamma_{j}-1} f(x) \mathrm{d} x \int_{x}^{\infty} y^{q-1}[\bar{F}(y)]^{\gamma_{j}-1} f(y) \mathrm{d} y .
\end{aligned}
$$

Proceeding on the lines of Theorem 3.1, the relation (30) yields on, using(26) and (12) and simplifications.

The relation (31) can be proved in view of [7] and using (4).

\section{Corollary 4}

When $m_{i}=m, i=1,2, \ldots, n-1$, relation (30) and (31) reduces to the product moment of $g$ os for Case-II.

$$
\begin{aligned}
& E\left[X^{p}(r, n, m, k) X^{q}(s, n, m, k)\right]-E\left[X^{p}(r, n, m, k) X^{q}(s-1, n, m, k)\right] \\
&=q \alpha C_{s-1} \sum_{i=1}^{r} \sum_{j=r+1}^{s} \sum_{l=0}^{\gamma_{j}} \sum_{c=0}^{\infty} \sum_{d=0}^{\infty} \sum_{t=0}^{\gamma_{i}-\gamma_{j}-1} \sum_{u=0}^{\infty}\left(\begin{array}{c}
r-1 \\
i
\end{array}\right)\left(\begin{array}{c}
s-r-1 \\
j
\end{array}\right) \\
& \times \frac{\left(\begin{array}{c}
\gamma_{j} \\
l
\end{array}\right)\left(\begin{array}{c}
\gamma_{i}-\gamma_{j}-1 \\
t
\end{array}\right)}{(m+1)^{s-2}(r-1) !^{2}} \frac{(\alpha c)_{d}(\alpha c-q)_{d}}{(\alpha c-q+1)_{d}} \times \frac{\lambda^{c+u+1} l^{c}(t+1)^{u}(-1)^{l+c+d+t+q+r+s-i-j}}{u ! d ! \beta^{p+q}\left(1-e^{-\lambda}\right)^{\gamma_{i}}(\alpha c-q)} \\
& \times \mathbf{B}(p+\alpha c-d+1 ; \alpha-\alpha u-p-q+\alpha c+d) .
\end{aligned}
$$

Also,

$$
\begin{aligned}
& E\left[X^{p}(r, n, m, k) X^{q}(s, n, m, k)\right]-E\left[X^{p}(r, n, m, k) X^{q}(s-1, n, m, k)\right] \\
& =\frac{q}{\gamma_{s} \alpha \beta \lambda} \sum_{a=0}^{\infty} \sum_{b=1}^{[\alpha(1-a)+1]}\left(\begin{array}{c}
{[\alpha(1-a)+1]} \\
b
\end{array}\right) \frac{\lambda^{a} \beta^{b}}{a !} E\left[X^{p}(r, n, m, k) X^{q+b-1}(s, n, m, k)\right] .
\end{aligned}
$$

Remark 3

Let $m_{i}=0, i=1,2, \ldots, n-1$ and $k=1$, then the recurrence relation for product moments of order statistics is

$$
E\left[X_{r: n}^{p} X_{s: n}^{q}\right]-E\left[X_{r: n}^{p} X_{s-1: n}^{q}\right]=\frac{q}{(n-s-1) \alpha \beta \lambda} \sum_{a=0}^{\infty} \sum_{b=1}^{[\alpha(1-a)+1]}\left(\begin{array}{c}
\alpha(1-a)+1] \\
b
\end{array}\right) \frac{\lambda^{a} \beta^{b}}{a !} E\left[X_{r: n}^{p} X_{s: n}^{q+b-1}\right] .
$$

Remark 4 When $m_{i} \rightarrow-1, i=1,2, \ldots, n-1$, the recurrence relation for product moments of $k^{\text {th }}$ upper record values will be

$$
\begin{aligned}
& E\left[\left(X_{U(r)}^{(k)}\right)^{p}\left(X_{U(s)}^{(k)}\right)^{q}\right]-E\left[\left(X_{U(r)}^{(k)}\right)^{p}\left(X_{U(s-1)}^{(k)}\right)^{q}\right] \\
& =\frac{q}{k \alpha \beta \lambda} \sum_{a=0}^{\infty} \sum_{b=1}^{[\alpha(1-a)+1]}\left(\begin{array}{c}
{[\alpha(1-a)+1]} \\
b
\end{array}\right) \frac{\lambda^{a} \beta^{b}}{a !} E\left[\left(X_{U(r)}^{(k)}\right)^{p}\left(X_{U(s)}^{(k)}\right)^{q+b-1}\right] .
\end{aligned}
$$




\section{Characterizations}

This section contains characterization results for the given distribution through recurrence relations for single and product moments of gos as well as through conditional moments.

\section{Theorem 5}

Fix a positive integer $k$ and let $p$ be a non-negative integer. A necessary and sufficient condition for a random variable $X$ to be distributed with $p d f$ given by (1) is that

$$
\begin{aligned}
& E\left[X^{p}(r, n, m, k)\right]-E\left[X^{p}(r-1, n, m, k)\right]= \\
& \frac{p}{\gamma_{r} \alpha \beta \lambda} \sum_{a=0}^{\infty} \sum_{b=1}^{[\alpha(1-a)+1]}\left(\begin{array}{c}
\alpha(1-a)+1] \\
b
\end{array}\right) \frac{\lambda^{a} \beta^{b}}{a !} E\left[X^{p+b-1}(r, n, m, k)\right] .
\end{aligned}
$$

Proof

The necessary part follows from (22). On the other hand, if the relation in (32) is satisfied, then on using [7], for $\xi(x)=x^{p}$, we have

$$
\begin{aligned}
\frac{p}{\gamma_{r}} \frac{C_{r-1}}{(r-1) !} & \int_{0}^{\infty} x^{p-1}[\bar{F}(x)]^{\gamma_{r}} g_{m}^{r-1}(F(x)) d x \\
= & \frac{p}{\gamma_{r} \alpha \beta \lambda} \frac{C_{r-1}}{(r-1) !} \int_{0}^{\infty} x^{p-1}[\bar{F}(x)]^{\gamma_{r-1}} g_{m}^{r-1}(F(x)) \\
& \times\left\{\sum_{a=0}^{\infty} \sum_{b=1}^{[\alpha(1-a)+1]}\left(\begin{array}{c}
{[\alpha(1-a)+1]} \\
b
\end{array}\right) \frac{\lambda^{a}(\beta x)^{b}}{a !} f(x)\right\} \mathrm{d} x
\end{aligned}
$$

or

$$
\begin{aligned}
& \frac{p}{\gamma_{r} \alpha \beta \lambda} \frac{C_{r-1}}{(r-1) !} \int_{0}^{\infty} x^{p-1}[\bar{F}(x)]^{\gamma_{r-1}} g_{m}^{r-1}(F(x)) \\
& \quad \times\left\{\alpha \beta \lambda \bar{F}(x)-\sum_{a=0}^{\infty} \sum_{b=1}^{[\alpha(1-a)+1]} \frac{\lambda^{a}(\beta x)^{b}}{a !}\left(\begin{array}{c}
\alpha(1-a)+1] \\
b
\end{array}\right) f(x)\right\}=0
\end{aligned}
$$

Applying the extension of Müntz-Szász theorem (see, for example, [16]) to (33), we get

$$
\bar{F}(x)=\frac{1}{\alpha \beta \lambda} \sum_{a=0}^{\infty} \sum_{b=0}^{[\alpha(1-a)+1)]} \frac{\lambda^{a}}{a !}\left(\begin{array}{c}
[\alpha(1-a)+1)] \\
b
\end{array}\right) \beta^{b} x^{b} f(x) .
$$

which proves the theorem.

\section{Theorem 6}

Fix a positive integer $k$ and let $p$ and $q$ be non-negative integers. A necessary and sufficient condition for a random variable $X$ to be distributed with $p d f$ given by (1) is

$$
\begin{aligned}
& E\left[X^{p}(r, n, m, k) X^{q}(s, n, m, k)\right]-E\left[X^{p}(r, n, m, k) X^{q}(s-1, n, m, k)\right] \\
& =\frac{q}{\gamma_{s} \alpha \beta \lambda} \sum_{a=0}^{\infty} \sum_{b=1}^{[\alpha(1-a)+1]}\left(\begin{array}{c}
{[\alpha(1-a)+1]} \\
b
\end{array}\right) \frac{\lambda^{a} \beta^{b}}{a !} E\left[X^{p}(r, n, m, k) X^{q+b-1}(s, n, m, k)\right] .
\end{aligned}
$$




\section{Proof}

The necessary part follows from (31). Now, suppose that the relation in (34) is satisfied. Then, using [7], for $\xi(x, y)=x^{p} y^{q}$, we have

$$
\begin{aligned}
& \frac{q}{\gamma_{s}} \frac{C_{s-1}}{(r-1) !(s-r-1) !} \int_{0}^{\infty} \int_{x}^{\infty} x^{p} y^{q-1}[\bar{F}(x)]^{m} f(x) g_{m}^{r-1}(x) \\
& \times\left[h_{m}(F(y))-h_{m}(F(x))\right]^{s-r-1}[\bar{F}(y)]^{\gamma_{s}} d y d x \\
&=\frac{q}{\gamma_{s} \alpha \beta \lambda} \frac{C_{s-1}}{(r-1) !(s-r-1) !} \int_{0}^{\infty} \int_{x}^{\infty} x^{p} y^{q-1}[\bar{F}(x)]^{m} f(x) g_{m}^{r-1}(x) \\
& \times\left[h_{m}(F(y))-h_{m}(F(x))\right]^{s-r-1}[\bar{F}(y)]^{\gamma_{s}-1} \\
& \times\left\{\sum_{a=0}^{\infty} \sum_{b=1}^{[\alpha(1-a)+1]} \frac{\lambda^{a}(\beta y)^{b}}{a !}\left(\begin{array}{c}
{[\alpha(1-a)+1]} \\
b
\end{array}\right) f(y)\right\} d y d x
\end{aligned}
$$

which implies,

$$
\begin{aligned}
& \frac{q}{\gamma_{s} \alpha \beta \lambda} \frac{C_{s-1}}{(r-1) !(s-r-1) !} \int_{0}^{\infty} \int_{x}^{\infty} x^{p} y^{q-1}[\bar{F}(x)]^{m} f(x) g_{m}^{r-1}(x) \\
& \times\left[h_{m}(F(y))-h_{m}(F(x))\right]^{s-r-1}[\bar{F}(y)]^{\gamma_{s}-1} \\
& \quad \times\left\{\alpha \beta \lambda \bar{F}(y)-\sum_{a=0}^{\infty} \sum_{b=1}^{[\alpha(1-a)+1]} \frac{\lambda^{a}(\beta y)^{b}}{a !}\left(\begin{array}{c}
{[\alpha(1-a)+1]} \\
b
\end{array}\right) f(y)\right\} d y d x=0 .
\end{aligned}
$$

Now applying the extension of Müntz-Szász theorem (see, for example, [16]) to (35), we get

$$
\bar{F}(y)=\sum_{a=0}^{\infty} \sum_{b=1}^{[\alpha(1-a)+1]} \frac{\lambda^{a}(\beta y)^{b}}{a !}\left(\begin{array}{c}
{[\alpha(1-a)+1]} \\
b
\end{array}\right) f(y),
$$

which proves the theorem.

\section{Theorem 7}

Let $X(r, n, m, k), r=1,2, \ldots, n$ be the the $r^{t h}$ gos based on continuous $d f F()$ and $E(X)$ exists. Then for two consecutive values $r$ and $r+1$, such that $1 \leq r<r+1 \leq n$,

$$
E\left[e^{-\lambda(1+\beta X(r+1, n, m, k))^{-\alpha}} \mid X(r, n, m, k)=x\right]=\frac{\gamma_{r+1} e^{-\lambda(1+\beta x)^{-\alpha}}+1}{\gamma_{r+1}+1}
$$

if and only if

$$
\bar{F}(x)=\frac{1-e^{-\lambda(1+\beta x)^{-\alpha}}}{1-e^{-\lambda}}, \quad x>0, \alpha>0, \beta>0, \lambda>0 .
$$

\section{Proof}

[21] have shown that

$$
E[h(X(s, n, m, k)) \mid X(r, n, m, k)=x]=a^{*} h(x)+b^{*}
$$

if and only if

$$
\bar{F}(x)=[a h(x)+b]^{c}
$$


with $a^{*}=\prod_{j=r+1}^{s}\left(\frac{c \gamma_{j}}{1+c \gamma_{j}}\right)$ and $b^{*}=-\frac{b}{a}\left(1-a^{*}\right)$.

Comparing (37) with (39), we get

$$
a=-\frac{1}{1-e^{-\lambda}}, h(x)=e^{-\lambda(1+\beta x)^{-\alpha}}, b=\frac{1}{1-e^{-\lambda}}, c=1 .
$$

Thus, the theorem can be proved in view of (38).

Corollary 5

For the $r^{t h}$ order statistics $X_{r: n}, r=1,2, \ldots n$ and under the condition as stated under Theorem 4.3

$$
E\left[e^{-\lambda\left(1+\beta X_{r+1: n}\right)^{-\alpha}} \mid X_{r: n}=x\right]=\frac{(n-r) e^{-\lambda(1+\beta x)^{-\alpha}}+1}{(n-r+1)}
$$

and consequently

$$
\begin{gathered}
E\left[e^{-\lambda\left(1+\beta X_{n: n}\right)^{-\alpha}} \mid X_{n-1: n}=x\right]=E\left[e^{-\lambda(1+\beta X)^{-\alpha}} \mid X \geq x\right] \\
=\frac{e^{-\lambda(1+\beta x)^{-\alpha}+1}}{2}
\end{gathered}
$$

if and only if

$$
\bar{F}(x)=\frac{1-e^{-\lambda(1+\beta x)^{-\alpha}}}{1-e^{-\lambda}}, x>0 ; \alpha, \beta, \lambda>0 .
$$

It may be noted that similar characterization result can also be seen for adjacent records as

$$
\begin{gathered}
E\left[e^{-\lambda\left(1+\beta X_{U(n)}\right)^{-\alpha}} \mid X_{U(n-1)}=x\right]= \\
=\left[e^{-\lambda(1+\beta X)^{-\alpha}} \mid X \geq x\right] \\
=\frac{e^{-\lambda(1+\beta x)^{-\alpha}+1}}{2} .
\end{gathered}
$$

\section{Conclusion}

The moments of ordered random variables and recurrence relations between them have received great attention in the past few years in statistical literature. We have obtained exact expressions and recurrence relations for single and product moments of generalized order statistics based on Poisson Lomax distribution. Since generalized order statistics is unified approach for several ordered random variables, thus results obtained can be easily deduced for order statistics, record values, sequential order statistics etc. Characterization theorems that use the properties of sample moments, order statistics, record statistics, and reliability properties can be applied to uniquely determine the associated stochastic model.

\section{Acknowledgement}

The authors of this manuscript are thankful to the learned referees for giving their fruitful suggestions in improving this script. The authors are also thankful to editor SOIC for giving their valuable time. 


\section{REFERENCES}

1. M. A. Ali, and A. H. Khan, Recurrence relations for the expectations of a function of single order statistic from general class of distributions, Journal of Indian Statistical Association, vol. 35, pp. 1-9, 1997.

2. M. A. Ali, and A. H. Khan, Recurrence relations for the expected values of certain functions of two order statistic, Metron, vol. 56, no. 1-2, pp. 1-9, 1998 .

3. B. Al-Zahranii, and H. Sagor, The Poisson-Lomax distribution, Revista Colombiana de Estadistica, vol. 37, no. 1, pp. 223-243, 2014.

4. Z. Anwar, H. Athar and R. U. Khan, Expectation identities based on recurrence relations of functions of generalized order statistics, Journal of Statistical Research, vol. 41, pp. 93-102, 2008.

5. B. C. Arnold, N. Balakrishnan and H. N. Nagaraja, A First Course in Order Statistics, John Wiley, New York., 1992.

6. H. Athar, and Z. Akhter, Some characterizations of continuous distributions based on order statistics, International Journal of Computational and Theoretical Statistics, vol. 2, no. 1, pp. 31-36, 2015.

7. H. Athar, and H. M. Islam, Recurrence relations for single and product moments of generalized order statistics from a general class of distributions, Metron., vol. 62, no. 3, pp. 327-337, 2004.

8. H. Athar, S. K. Khwaja and Nayabuddin, Expectation identities of Pareto distributions based on generalized order statistics and its characterization, American Journal of Applied Mathematics and Mathematical Sciences, vol. 1, no. 1, pp. 23-29, 2012.

9. H. Athar and Z. Noor, Characterization of probability distributions through conditional expectation of function of pair of order statistics, Journal of Applied Probability and Statistics, vol. 8, no. 1, pp. 45-56, 2013.

10. H. Athar and Y. A. Aty, Characterization of general class of distribution by truncated moment, Thailand Statistician, vol. 18, no. 2 , in press, 2020

11. H. Athar, M. Yaqub and H. M. Islam, On characterizations of distributions through linear regression of record values and order statistics, Aligarh Journal of Statistics, vol. 23, 97-105, 2003.

12. N. Balakrishnan, H. J. Malik and S. E. Ahmed, Recurrence relations and identities of moments of order statistics, II: Specific continuous distributions, Communications in Statistics-Theory and Methods, vol. 17, no. 8, 2657-2694, 1988.

13. J. Galambos and S. Kotz, Characterizations of Probability Distributions, Springer, New York, 1978.

14. I. S. Gradshteyn and I. M. Ryzhik, Tables of Integrals, Series and Products, Edited by Jeffrey, A. and Zwillinger, D. 7th Ed., Academic Press, New York, 2007.

15. N. Gupta and Z. Anwar, Relations for Single and Product Moments of Odds Generalized Exponential-Pareto Distribution Based on Generalized Order Statistics and Characterization, Statistics, Optimization and Information Computing, vol. 7, no. 1,160C170, 2019.

16. J. S. Hwang and G. D. Lin, Extentions of Müntz-Szász theorems and applications, Analysis, vol. 4, 143-160, 1984.

17. U. Kamps, A Concept of Generalized Order Statistics, B.G. Tuebner Stuttgart, Germany, 1995.

18. U. Kamps and E. Cramer, On distribution of generalized order statistics, Statistics, vol. 35, no. 3, 269-280, 2001.

19. C. Keseling, Conditional distributions of generalized order statistics and some characterizations, Metrika, vol. 49, 27-40, 1999.

20. A. H. Khan and A. M. Abouammoh, Characterization of distributions by conditional expectation of order statistics, Journal of Applied Statistics and Science, vol. 13, no. 2, 159-167, 2000.

21. A. H. Khan and A. A. Alzaid, Characterization of distributions by conditional expectation of order statistics, Journal of Applied Statistics and Science, vol. 13,123-136, 2004.

22. A. H. Khan and H. Athar, Characterization of distributions through order statistics, Journal of Applied Statistics and Science, vol 13, no. 2, 159-167, 2004.

23. A. H. Khan, R. U. Khan and M. Yaqub, Characterization of continuous distributions through conditional expectation of function of generalized order statistics, Journal of Applied Probability and Statistics, vol. 1, no. 1, 115-131, 2006.

24. A. H. Khan, S. Parvez and M. Yaqub, Recurrence relations between product moments of order statistics, Journal of Statisticsn Planning and Inference, vol. 8, 175-183, 1983.

25. A. H. Khan, S. Parvez and M. Yaqub, Recurrence relations between moments of order statistics, Naval Research Logistics Quarterly, vol. 30, no. 3, 419-441, 1983.

26. R. U. Khan, M. A. Khan and M. A. R. Khan, Relations for moments of generalized record values from additive Weibull distribution and associated inference, Statistics, Optimization and Information Computing, vol. 5, no. 2, 127-136, 2017.

27. R. U. Khan, D. Kumar and H. Athar, Moments of generalized order statistics from Erlang-truncated exponential distribution and its characterization, International Journal of Statistics and Systems, vol. 5, no. 4, 455-464, 2010.

28. S. Kotz and D. N. Shanbhag, Some new approaches to probability distributions, Advances in Applied Probability, vol. 12, no. 4, 455-464, 1980

29. H. J. Malik, N. Balakrishnan and S. E. Ahmed, Recurrence relations and identities of moments of order statistics, I: Arbitrary continuous distributions, Communications in Statistics - Theory and Methods, vol. 17, 2623-7826, 1988.

30. Nayabuddin and H. Athar, Recurrence relations for single and product moments of generalized order statistics from Marshall-Olkin extended Pareto distribution, Communications in Statistics - Theory and Methods, vol. 46, no. 16, 7820-7826, 2017.

31. P. Pawlas and D. Szynal, Recurrence relations for single and product moments of generalized order statistics from Pareto, Generalized Pareto and Burr distributions, Communications in Statistics - Theory and Methods, vol. 30, no. 4, 739-746, 2001.

32. B. Singh, R. U. Khan and M. A. R. Khan, Generalized order statistics from Kumaraswamy-Burr III distribution and related inference, Journal of Statistics: Advances in Theory and Applications, vol. 19, no. 1, 1-16, 2018. 